\title{
New limits on Magnetic Monopoles searches from accelerator and non-accelerator experiments
}

\author{
Michela $\operatorname{Cozzi}^{1, *}$ \\ ${ }^{1}$ Dipartimento di Fisica dell'Università di Bologna and INFN Sezione di Bologna, \\ Viale B. Pichat 6/2, I-40127, Bologna, Italy \\ Invited talk at V International Conference on Non-Accelerator New Physics \\ Dubna, Russia, June 2005
}

\begin{abstract}
Here the status of the searches for "classical Dirac" Magnetic Monopoles (MMs) at accelerators and for GUT MMs in the cosmic radiation is discussed. We present recent analysis for "classical Dirac" monopoles at accelerators and the lowest flux upper limit for Magnetic Monopoles in the mass range $10^{5}-10^{12} \mathrm{GeV}$ obtained with the SLIM experiment at the Chacaltaya High Altitude Laboratory (5290 m a.s.l.).
\end{abstract}

\section{INTRODUCTION}

The lack of symmetry between electric and magnetic charges is one of the oldest puzzles in physics. Why is it possible to isolate positive and negative electric charges, but not north and south magnetic poles? At the beginning of the 19th century there were discussions concerning the magnetic content of matter and the possible existence of isolated magnetic charges. While the existence of magnetic monopoles is not excluded by classical electromagnetism, the first convincing argument in favor of such particles was made by Dirac in 1931 [1]. Dirac linked the existence of magnetic monopoles with the quantization of electric charge and established the relation between the elementary electric charge $e$ and a basic magnetic charge $g$ :

$$
e \cdot g=\frac{n \hbar c}{2}=n g_{D}, \quad n=1,2, \ldots
$$

\footnotetext{
* Michela.Cozzi@bo.infn.it; We acknowledge the cooperation of the members of the SLIM Collaboration. We thank our collaborators and many colleagues for discussions and advise, in particular S. Balestra, S. Cecchini, G. Giacomelli and L. Patrizii
} 
where $\mathrm{n}$ is an unknow integer and $g_{D}=\hbar c / 2 e=68.5 e$ is the unit Dirac charge. Here we assume that the elementary electric charge is that of an electron. If free quarks exist, Eq. 1 should be modified by replacing $e$ with $e / 3$, which effectively increases $g$ by a factor of 3 . The existence of magnetic charges and of magnetic currents would symmetrize in form Maxwell's equations. The symmetry would not be perfect since $e \neq g$ but the couplings could be energy dependent and could merge in a common value at high energies [2]. There was no prediction for the MM mass; a rough estimate, obtained assuming that the classical monopole radius is equal to the classical electron radius yields $m_{M} \approx g^{2} m_{e} / e^{2} \approx n \cdot 4700 m_{e} \approx n \cdot 2.4 \mathrm{GeV} / \mathrm{c}^{2}$. From 1931 searches for "classical Dirac" monopoles were carried out at every new high-energy accelerator employing a variety of direct and indirect methods [3].

Interest in monopoles revived in the 70's, following the discovery by 't Hooft and Polyakov [4] that there exist monopole solutions to the field equations of theories in which a semisimple unifying gauge group breaks into a $\mathrm{U}(1)$ group plus others. If the unification scale is $M_{G U T}$, the monopole mass [5] is $m_{M} \geq M_{G U T} / \alpha_{G U T}$, with $\alpha_{G U T}$ the grand-unified coupling constant. A consequence of these theoretical developments is that monopoles are expected to be extremely heavy. In GUTs with $M_{G U T} \simeq 10^{14}-10^{15} \mathrm{GeV}$ and $\alpha_{G U T} \simeq 0.025, m_{M}>$ $10^{16}-10^{17} \mathrm{GeV}$. This is an enormous mass: MMs cannot be produced at any man-made accelerator, existing or conceivable. They could only be produced in the first instants of our Universe and can be searched for in the penetrating Cosmic Radiation (CR). The most stringent direct limits on GUT superheavy MMs have been set by the MACRO experiment [6].

Larger MM masses are expected if gravity is brought into the unification picture, and in some SuperSymmetric models.

Intermediate mass monopoles (IMMs) may have been produced in later phase transitions in the Early Universe, in which a semisimple gauge group yields a U(1) group [7]. IMMs with masses $10^{5} \div 10^{12} \mathrm{GeV}$ may be accelerated to relativistic velocities in the galactic magnetic field, and in several astrophysical sites. It has been speculated that very energetic IMMs could yield the highest energy cosmic rays [8].

The lowest mass MM should be stable, since magnetic charge is conserved like electric charge. Therefore, the MMs produced in the Early Universe should still exist as cosmic relics, whose kinetic energy has been affected first by the expansion of the Universe and then by their travel through galactic and intergalactic magnetic fields. 
Here we shall review the present experimental situation on MM searches with emphasis on the recent analysis for "classical Dirac" monopoles at accelerators and on recent limits for Intermediate Mass MMs searches with the SLIM experiment [9].

\section{PROPERTIES OF MAGNETIC MONOPOLES}

The main properties of MMs are obtained from the Dirac relation.

- If $n=1$ and the basic electric charge is that of the electron, then the basic magnetic charge is $g_{D}=\hbar c / 2 e=137 e / 2$. The magnetic charge is larger if $n>1$ and if the basic electric charge is $e / 3$.

- In analogy with the fine structure constant, $\alpha=e^{2} / \hbar c \simeq 1 / 137$, the dimensionless magnetic coupling constant is $\alpha_{g}=g_{D}^{2} / \hbar c \simeq 34.25$; since it is $>1$ perturbative calculations cannot be used.

- Energy $W$ acquired in a magnetic field $B: W=n g_{D} B \ell=n 20.5 \mathrm{keV} / \mathrm{G} \mathrm{cm}$. In a coherent galactic-length $(\ell \simeq 1 \mathrm{kpc}, B \simeq 3 \mu \mathrm{G})$, the energy gained by a MM with $g=g_{D}$ is $W \simeq 1.8 \times 10^{11} \mathrm{GeV}$. Classical poles and IMMs in the CR may be accelerated to relativistic velocities. GUT poles should have low velocities, $10^{-4}<\beta<10^{-1}$.

- MMs may be trapped in ferromagnetic materials by an image force, which could reach values of $\sim 10 \mathrm{eV} / \AA$.

- The interaction of a MM magnetic charge with a nuclear magnetic dipole could lead to the formation of a M-nucleus bound system. A monopole-proton bound state may be produced via radiative capture. Monopole-nucleus bound states may exist for nuclei with large gyromagnetic ratios.

- Energy losses of fast poles. A fast MM with magnetic charge $g_{D}$ and velocity $v=\beta c$ behaves like an electric charge $(z e)_{e q}=g_{D} \beta$, Fig. 1 .

- Energy losses of slow poles $\left(10^{-4}<\beta<10^{-2}\right)$ may be due to ionization or excitation of atoms and molecules of the medium ("electronic" energy loss) or to recoiling atoms or nuclei ("atomic" or "nuclear" energy loss). Electronic energy loss predominates for $\beta>10^{-3}$.

- Energy losses at very low velocities. MMs with $v<10^{-4}$ c may lose energy in elastic collisions with atoms or with nuclei. The energy is released to the medium in the form of elastic vibrations and/or infra-red radiation [10].

Fig. 1 shows the energy loss in liquid hydrogen of a $g=g_{D}$ MM vs $\beta$ [11]. 


\section{SEARCH FOR "CLASSICAL DIRAC" MONOPOLES AT ACCELERATORS}

By classical monopole we mean a particle without electric charge or hadronic interactions and with magnetic charge $g$ satisfying the Dirac quantization condition (Eq. 1). Monopole searches at accelerators have predominantly used either induction or ionization methods.

Induction experiments measure the monopole magnetic charge and are independent of monopole mass and velocity. The method of detection is the search for the induction of a persistent current within a superconducting loop [12]. Searches for magnetic monopoles using this method have been performed at the $p \bar{p}$ Tevatron collider assuming that produced MMs could stop, be trapped and bound in the matter surrounding the D0 and CDF collision regions [13]. Pieces of the detector materials were cut into long thin strips which were each passed through a superconducting coil coupled to a Superconducting QuantumMechanical Interference Device (SQUID). Trapped magnetic monopoles in a strip will cause a persistent current to be induced in the superconducting coil by the magnetic field of the monopole, after complete passage of the strip through the coil. In contrast, the current due to magnetic dipoles returns to zero after passage of the strip.

Ionization experiments rely on a magnetic charge producing more ionization than an electrical charge with the same velocity. Direct searches for magnetic monopoles using different tracking devices as scintillators, nuclear track detectors (NTDs) or central detectors of complex experiments, were performed at $p p, p \bar{p}$ and $\mathrm{e}^{+} \mathrm{e}^{-}$colliders. Experiments at Tevatron established cross section limits of $\sim 2 \times 10^{-34} \mathrm{~cm}^{2}$ for MMs with $m_{M}<850 \mathrm{GeV}$ [14], searches LEP excluded masses up to $45 \mathrm{GeV}$ [15].

Indirect searches for classical monopoles have looked for the effects of virtual monopole/anti-monopole loops added to QED Feynman diagrams in $\bar{p} p$ and $\mathrm{e}^{+} \mathrm{e}^{-}$collisions. An indirect search for MMs is the search for multi- $\gamma$ events. Five peculiar photon showers found in emulsion plates exposed to high-altitude CRs, are characterized by an energetic narrow cone of tens of photons, without any incident charged particle [16]. The total energy of the photons is $\sim 10^{11} \mathrm{GeV}$. The small radial spread of photons suggested a c.m. $\gamma=\left(1-\beta^{2}\right)^{-1 / 2}>10^{3}$. The energies of the photons are too small to have $\pi^{o}$ decays as their source. One possible explanation: a high-energy $\gamma$-ray, with energy $>10^{12} \mathrm{eV}$, produced a pole-antipole pair, which suffered bremsstrahlung and annihilation producing the final multi- $\gamma$ events. Searches for multi- $\gamma$ events were performed at the Tevatron and LEP 
colliders (Fig. 2). The D0 experiment searched for $\gamma$ pairs with high transverse energies; virtual pointlike MMs may rescatter pairs of nearly real photons into the final state via a box monopole diagram; they set a $95 \% \mathrm{CL}$ limit of $870 \mathrm{GeV}$ [17]. At LEP the L3 coll. searched for $Z \rightarrow \gamma \gamma \gamma$ events; no deviation from QED predictions was observed, setting a 95\% CL limit of $510 \mathrm{GeV}$ [18]. Many authors studied the effects from virtual monopole loops [2, 19]. Since the Standard Model $Z^{0}$-boson could couple to monopoles, assuming that the coupling between the $Z^{0}$ and a MM pair is larger than for any lepton pair, the measurement of the $Z^{0}$ decay width provides an indirect limit on MMs production for $m_{M}<m_{Z} / 2$ [2].

Fig. 2 summarizes the cross section limits vs MM mass obtained by direct and indirect experiments (at the Fermilab $\bar{p} p$ collider, $\mathrm{e}^{+} \mathrm{e}^{-}$colliders, the ISR $p p$ collider [11]. Most searches are sensitive to poles with magnetic charges $g=n g_{D}$ with $0.5<n<5$.

Recently, new limits on Magnetic Monopoles searches have been carried out from OPAL and $\mathrm{H} 1$ Collaborations. A new direct search for MM pairs produced in the reaction $\mathrm{e}^{+} \mathrm{e}^{-} \rightarrow$ $M \bar{M}(\gamma)$ have been performed by the OPAL collaboration at LEP2. This search is primarily based on the $d E / d x$ in the tracking chamber of the OPAL detector [21]: due to their large velocities these particles would have high ionization energy losses. This analysis is sensitive to MMs with masses from $45 \mathrm{GeV}$ up to the kinematic limit (about $103 \mathrm{GeV}$ ).

The first search for MMs in $e^{+} p$ collisions, at a centre of mass energy of $300 \mathrm{GeV}$, was made by the $\mathrm{H} 1$ collaboration at HERA [22]. This analysis assumed that heavily ionizing MMs produced in $e^{+} p$ collisions may stop in the beam pipe surrounding the H1 interaction point at HERA. The binding energy of monopoles in the material is expected to be large and so they should remain permanently trapped providing they are stable. The beam pipe surrounding the interaction region during 1995-1997 (integrated luminosity $60 \mathrm{pb}^{-1}$ ) was investigated using a SQUID magnetometer with a sensitivity of $0.2 g_{D}$ to look for stopped magnetic monopoles. No free magnetic charges were observed and charge-dependent upper limits on the cross section for the electro-production of magnetic monopoles have been set.

\section{SEARCH FOR GUT MONOPOLES IN THE COSMIC RADIATION}

As already stated, GUT theories of the electroweak and strong interations predict the existence of superheavy MMs produced in the Early Universe (EU) when the GUT gauge group breaks into separate groups, one of which is U(1). Assuming that the GUT group 
is $\mathrm{SU}(5)$ (which is excluded by proton decay experiments) one should have the following transitions:

$$
\begin{aligned}
& 10^{15} \mathrm{GeV} \quad 10^{2} \mathrm{GeV} \\
& \begin{array}{cccc}
S U(5) \longrightarrow & S U(3)_{C} \times\left[S U(2)_{L} \times U(1)_{Y}\right] & \longrightarrow & S U(3)_{C} \times U(1)_{E M} \\
& 10^{-35} s & 10^{-9} s &
\end{array}
\end{aligned}
$$

MMs would be generated as topological point defects in the GUT phase transition, almost one pole for each causal domain. In the standard cosmology this leads to too many poles (the monopole problem). Inflation would defer the GUT phase transition after large supercooling; in its simplest version the number of generated MMs would be very small. However the flux depends critically on several parameters, like the pole mass, the reheating temperature, etc. If the reheating temperature is large enough one would have MMs produced in high energy collisions, like $\mathrm{e}^{+} \mathrm{e}^{-} \rightarrow M \bar{M}$.

A flux of cosmic GUT MMs may reach the Earth with a velocity spectrum in the range $4 \times 10^{-5}<\beta<0.1$, with possible peaks corresponding to the escape velocities from the Earth, the Sun and the Galaxy. Searches for such MMs in the CR performed with superconducting induction devices yielded a combined $90 \%$ CL limit of $2 \times 10^{-14} \mathrm{~cm}^{-2} \mathrm{~s}^{-1} \mathrm{sr}^{-1}$, independent of $\beta$ [11]. Direct searches were performed above ground and underground. MACRO performed a search with different types of detectors (liquid scintillators, limited streamer tubes and NTDs) with an acceptance of $\sim 10,000 \mathrm{~m}^{2} \mathrm{sr}$ for an isotropic flux. No MM was detected; the 90\% CL flux limits, shown in Fig. 3 vs $\beta$ for $g=g_{D}$, are at the level of $1.4 \times 10^{-16} \mathrm{~cm}^{-2} \mathrm{~s}^{-1} \mathrm{sr}^{-1}$ for $\beta>4 \times 10^{-5}[6]$. The figure shows also the limits from the Ohya [23], Baksan, Baikal, and AMANDA experiments [24].

\section{THE SLIM EXPERIMENT}

The SLIM experiment, which searches for IMMs with NTDs at the Chacaltaya high altitude lab (5290 m a.s.l.), is sensitive to $g=2 g_{D}$ MMs in the whole range $4 \times 10^{-5}<\beta<1$ [9].

The SLIM apparatus at Chacaltaya consists of $440 \mathrm{~m}^{2}$ of CR39 and Makrofol nuclear track detectors. The installation began in March 2000 and was completed in July 2001. Further $100 \mathrm{~m}^{2}$ were installed at Koksil (Himalaya) since 2003. The detector is organized in modules of $24 \mathrm{~cm}$ x $24 \mathrm{~cm}$, each made of 3 layers of CR39 (1.4 mm thick), 3 layers of 
polycarbonate (Makrofol, $0.5 \mathrm{~mm}$ thick) and of an aluminium absorber (1 $\mathrm{mm}$ thick); each module is sealed in an aluminized plastic bag filled with dry air.

About $226 \mathrm{~m}^{2}$ of CR39 have been etched and analysed, with an average exposure time of 3.6 years. No candidate passed the searching criteria: the $90 \%$ C.L. flux upper limits for fast $(\beta>0.1)$ IMMTs coming from above, are at the level of $2.910^{-15} \mathrm{~cm}^{-2} \mathrm{sr}^{-1} \mathrm{~s}^{-1}$.

\section{CONCLUSIONS}

Searches for MMs have shown a remarkable progress since 1931. This is particularly impressive, since no direct evidence of the existence of monopoles has been found. Even without such evidence, strong theoretical motivations continue to legitimate the experimental program in this field.

Direct and indirect accelerator searches for "classical Dirac" MMs placed limits at the level $m_{M}>850 \mathrm{GeV}$ with cross section upper values as shown in Fig. 2. Future improvements may come from experiments at Fermilab collider and at the future LHC.

Many searches were performed for heavy GUT MMs in the penetrating cosmic radiation. The $90 \%$ CL flux limits are at $\sim 1.4 \times 10^{-16} \mathrm{~cm}^{-2} \mathrm{~s}^{-1} \mathrm{sr}^{-1}$ for $\beta \geq 4 \times 10^{-5}$. It may be difficult to do much better since one would require refined detectors of considerably larger areas.

Present limits on Intermediate Mass Monopoles with high $\beta$ are relatively poor. By the end of 2006 the SLIM analysis will be completed and the experiment will reach a sensitivity of $10^{-15} \mathrm{~cm}^{-2} \mathrm{sr}^{-1} \mathrm{~s}^{-1}$ for $\beta \geq 10^{-2}$ and IMMs with $10^{7}<m_{I M M}<10^{13} \mathrm{GeV}$. Moreover this search will benefit from the analysis of further $100 \mathrm{~m}^{2}$ of NTDs installed at Koksil (Pakistan).

1. P. A. M. Dirac, Proc. R. Soc. London 133 (1931) 60; Phys. Rev. 74 (1948) 817.

2. A. De Rujula, Nucl. Phys. B435 (1995) 257.

3. G. Giacomelli, Riv. Nuovo Cimento 7 N.12 (1984) 1.

4. G. 't Hooft, Nucl. Phys. B79 (1974) 276; A. M. Polyakov, JETP Lett. 20 (1974) 194.

5. E. B. Bogomolny, Sov. J. Nucl. Phys. 24 (1976) 801; L. D. Fadeev, Lett. Math. Phys. 1 (1976) 289; S. Coleman et al., Phys. Rev. D15 (1977) 544. 
6. M. Ambrosio et al., Eur. Phys. J. C25 (2002) 511; M. Ambrosio et al., Eur. Phys. J. C26 (2002) 163.

M. Ambrosio et al., Nucl. Instr. Meth. A486 (2002) 663.

7. G. Lazarides et al., Phys. Rev. Lett. 58 (1987) 1707; T. W. Kephart and Q. Shafi, Phys. Lett. B520 (2001) 313.

8. P. Bhattacharjee et al., Phys. Rept. 327 (2000) 109 and refs. therein.

9. S. Balestra et al., hep-ex/0508043; S. Cecchini et al., hep-ex/0503003; hep-ex/0502034.

10. J. Derkaoui et al., Astrop. Phys. 9 (1998) 173; Astrop. Phys. 9 (1999) 339.

11. G. Giacomelli and L. Patrizii hep-ex/0112009; hep-ex/0302011; hep-ex/0506014.

12. L.W. Alvarez et al., Science 167 (1970) 701.

13. G.R. Kalbfleisch et al., Phys. Rev. Lett. 85 (2000) 5292.

14. M. Bertani et al., Europhys. Lett. 12 (1990) 613.

15. K. Kinoshita et al., Phys. Rev. D46 (1992) R881.

16. M. Schein et al., Phys. Rev. 99 (1955) 643.

17. B. Abbott et al., Phys. Rev. Lett. 81 (1998) 524.

18. M. Acciarri et al., Phys. Lett. B345 (1995) 609.

19. I. F. Ginzburg and A. Schiller, Phys. Rev. D60 (1999) 075016.

20. L. Gamberg et al., hep-ph/9906526.

21. K. Ahmet et al., OPAL Collab., Nucl. Instr. Meth. A305 (1991) 275; G. Abbiendi et al., Phys. Lett. $\mathbf{B}$ (to be published).

22. A. Aktas et al., H1 Collab., Eur. Phys. J. C41 (2005) 133.

23. S. Orito et al. ("Ohya"), Phys. Rev. Lett. 66 (1991) 1951.

24. E. N. Alexeyev et al. ("Baksan"), 21 15t ICRC 10 (1990) 83; V. A. Balkanov et al. ("Baikal") Nucl. Phys. B(Proc. Suppl.) 91 (2001) 438; P. Niessen et al., 2ryt ICRC 3 (2001) 1496. 


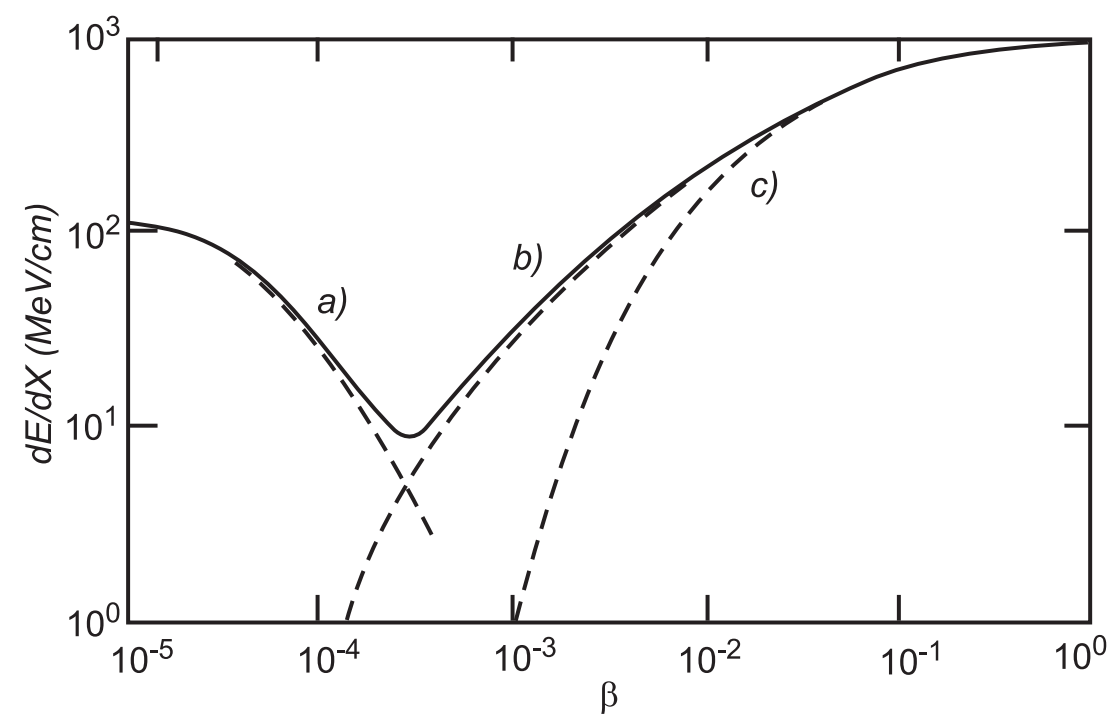

Figure 1. The energy losses, in $\mathrm{MeV} / \mathrm{cm}$, of $g=g_{D} \mathrm{MMs}$ in liquid hydrogen vs $\beta$. Curve a) corresponds to elastic monopole-hydrogen atom scattering; curve b) to interactions with level crossings; curve c) describes the ionization energy loss.

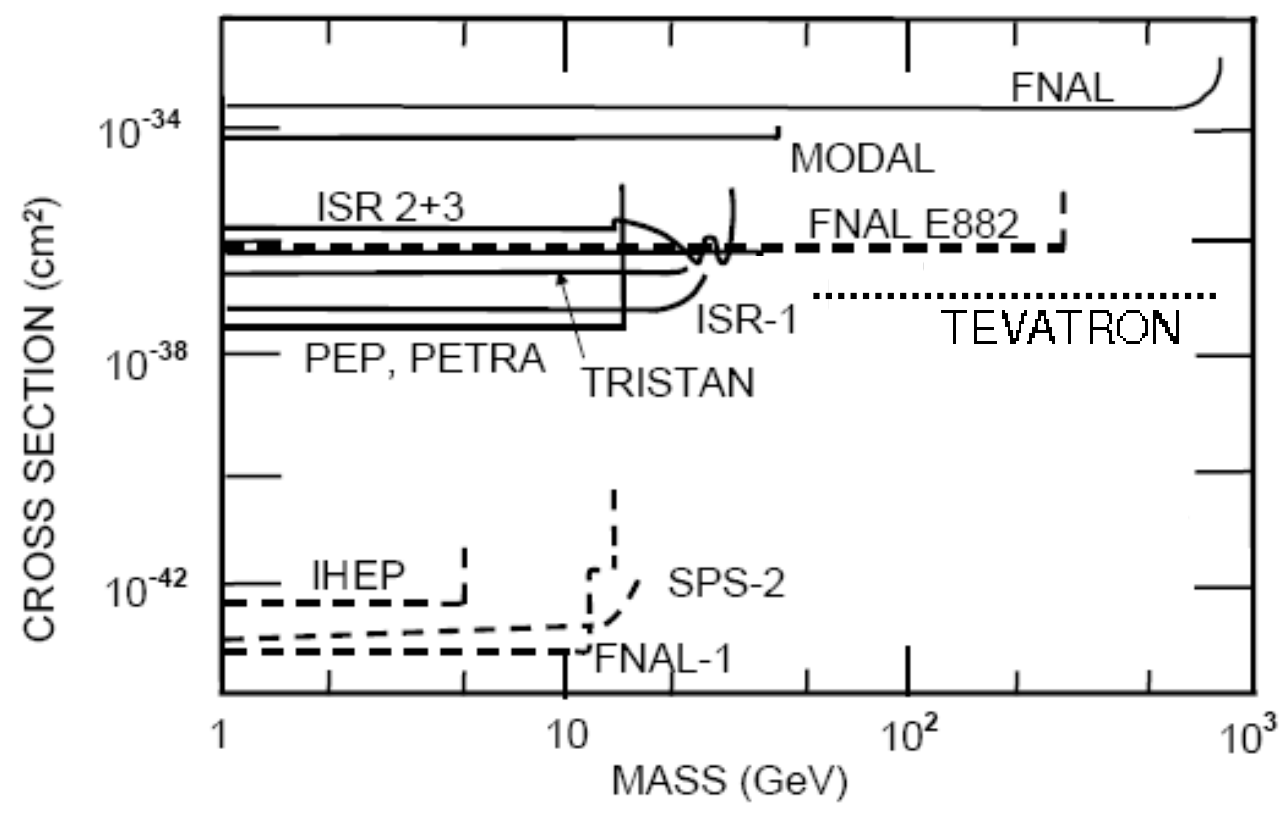

Figure 2. "Classical Dirac" MMS cross section upper limits vs MM mass obtained from direct and indirect accelerator searches. (solid lines: searches performed with tracking device, dashed lines: other methods. 


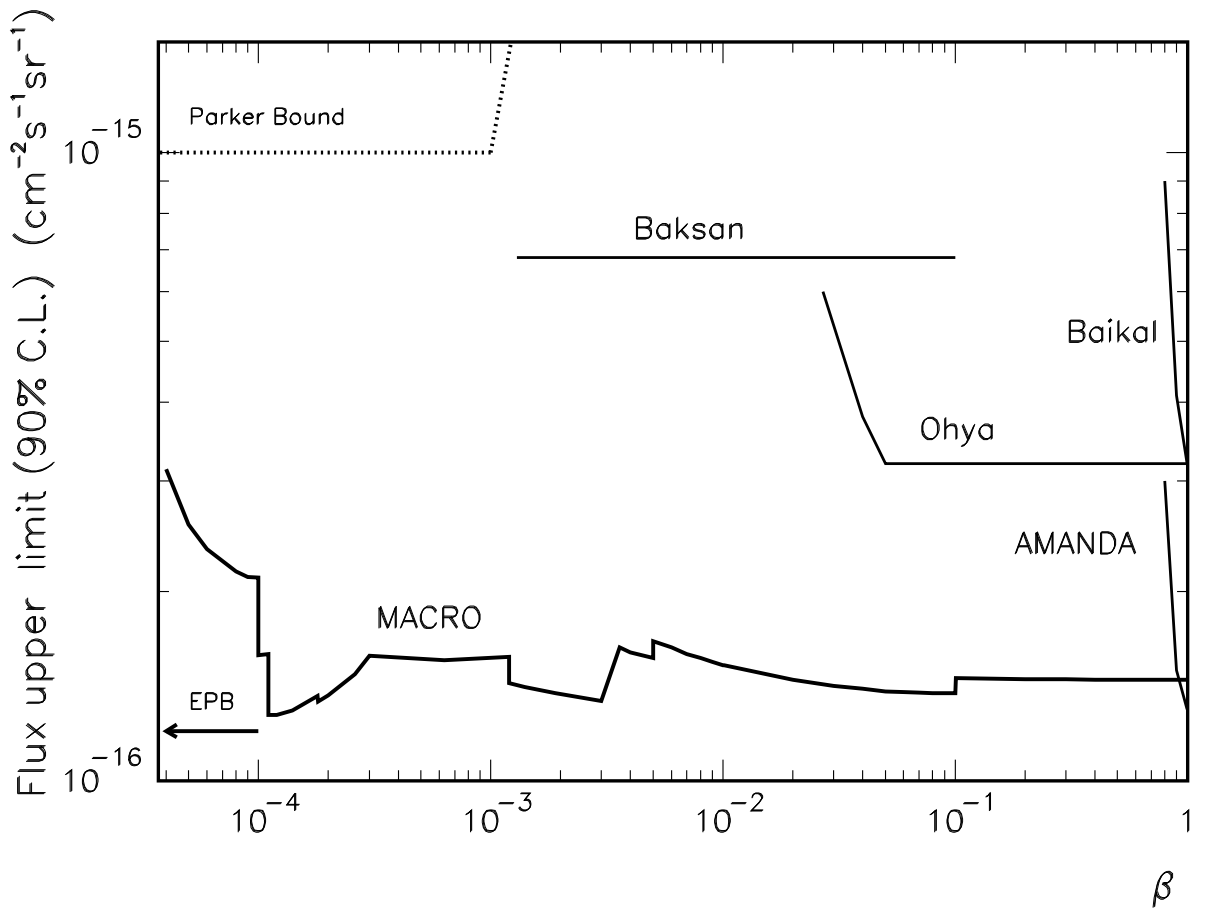

Figure 3. The $90 \%$ CL MACRO direct upper limits vs $\beta$ for GUT $g=g_{D}$ poles in the penetrating $\mathrm{CR}$, and direct limits from other experiments (see text). 